\title{
Stability and Bifurcation Analysis on an Ecoepidemiological Model with Stage Structure and Time Delay
}

\author{
Lingshu Wang ${ }^{1}$ and Guanghui Feng ${ }^{2}$ \\ ${ }^{1}$ School of Mathematics and Statistics, Hebei University of Economics \& Business, Shijiazhuang 050061, China \\ ${ }^{2}$ Institute of Applied Mathematics, Shijiazhuang Mechanical Engineering College, Shijiazhuang 050003, China \\ Correspondence should be addressed to Lingshu Wang; wanglingshu@126.com
}

Received 15 April 2014; Accepted 21 July 2014; Published 12 August 2014

Academic Editor: Sanling Yuan

Copyright (c) 2014 L. Wang and G. Feng. This is an open access article distributed under the Creative Commons Attribution License, which permits unrestricted use, distribution, and reproduction in any medium, provided the original work is properly cited.

\begin{abstract}
An ecoepidemiological predator-prey model with stage structure for the predator and time delay due to the gestation of the predator is investigated. The effects of a prey refuge with disease in the prey population are concerned. By analyzing the corresponding characteristic equations, the local stability of each of the feasible equilibria of the model is discussed. Further, it is proved that the model undergoes a Hopf bifurcation at the positive equilibrium. By means of appropriate Lyapunov functions and LaSalle's invariance principle, sufficient conditions are obtained for the global stability of the semitrivial boundary equilibria. By using an iteration technique, sufficient conditions are derived for the global attractiveness of the positive equilibrium.
\end{abstract}

\section{Introduction}

In the natural world, species does not exist alone. While species spreads the disease, it also competes with the other species for space or food, or it is predated by other species. The construction and study of models for the population dynamics of predator-prey systems have been an important topic in theoretical ecology. Following Anderso and May [1], who were the first to propose an ecoepidemiological model by merging the ecological predator-prey model introduced by Lotka and Volterra, the effect of disease in ecological system is an important issue from mathematical and ecological point of view. Ecoepidemiology which is a relatively new branch of study in theoretical biology tackles such situations by dealing with both ecological and epidemiological issues.

The research of the hiding behaviour of preys has been incorporated as a new ingredient of predator-prey models. In nature, prey populations often have access to areas where they are safe from their predators. Such refugia are usually playing two significant roles, serving both to reduce the chance of extinction due to predation and to damp predatorprey oscillations. It is well known that many more attentions have been paid on the effects of a prey refuge for predatorprey model. In [2], Wang considered an ecoepidemiological model incorporating a prey refuge with disease in the prey population

$$
\begin{gathered}
\frac{d S}{d t}=r S(t)\left(1-\frac{S(t)+I(t)}{K}\right)-\beta S(t) I(t) \\
-b_{1}(1-m) S(t) Y(t), \\
\frac{d I}{d t}=\beta S(t) I(t)-b_{2}(1-m) I(t) Y(t)-d I(t), \\
\frac{d Y}{d t}=p b_{1}(1-m) S(t) Y(t) \\
+p b_{2}(1-m) I(t) Y(t)-c Y(t),
\end{gathered}
$$

where $S(t)$ and $I(t)$ represent the densities of susceptible and infected prey population at time $t$, respectively, and $Y(t)$ represents the density of the predator population at time $t$. The parameters $r, K, \beta, b_{1}, b_{2}, c, d$, and $p$ are positive constants in which $r$ and $K$ represent the prey intrinsic growth rate and the carrying capacity, respectively. $\beta$ is the transmission rate of the susceptible prey into the infected prey. $b_{1}$ and $b_{2}$ are the capturing rates of the susceptible prey and the infected prey, respectively. $p$ describes the efficiency of the predator in converting consumed prey into predator offspring. The 
constant proportion infected prey refuge is $(1-m) I$, where $m \in[0,1)$ is a constant. By means of appropriate Lyapunov functions and limit theory, sufficient conditions are obtained for the global stability of the semitrivial boundary equilibria of model (1).

We note that it is assumed in system (1) that each individual predator admits the same ability to feed on prey. This assumption seems to be not realistic for many animals. In the natural world, there are many species whose individuals pass through an immature stage during which they are raised by their parents, and the rate at which they attack prey can be ignored. Moreover, it can be assumed that their reproductive rate during this stage is zero. Stage structure is a natural phenomenon and represents, for example, the division of a population into immature and mature individuals. Stagestructured models have received great attention in recent years (see, e.g., [3-5]).

Time delays of one type or another have been incorporated into biological models by many researchers (see, e.g., [5-7]). In general, delay differential equations exhibit much more complicated dynamics than ordinary differential equations since a time delay could cause the population to fluctuate. Time delay due to gestation is a common example, because generally the consumption of prey by the predator throughout its past history governs the present birth rate of the predator. Therefore, more realistic models of population interactions should take into account the effect of time delays.

Based on the above discussions, in this paper, we incorporate a stage structure for the predator and time delay due to the gestation of predator into the model (1). To this end, we study the following differential equations:

$$
\begin{gathered}
\frac{d S}{d t}=r S(t)\left(1-\frac{S(t)+I(t)}{K}\right)-\beta S(t) I(t), \\
\frac{d I}{d t}=\beta S(t) I(t)-d I(t)-b(1-m) I(t) Y_{2}(t), \\
\frac{d Y_{1}}{d t}=p b(1-m) I(t-\tau) Y_{2}(t-\tau)-\left(r_{1}+d_{1}\right) Y_{1}(t), \\
\frac{d Y_{2}}{d t}=r_{1} Y_{1}(t)-d_{2} Y_{2}(t)-a Y_{2}^{2}(t),
\end{gathered}
$$

where $Y_{1}(t)$ and $Y_{2}(t)$ represent the densities of the immature and the mature predator population at time $t$, respectively. The parameters $d_{1}, d_{2}$, and $r_{1}$ are positive constants in which $d_{1}$ and $d_{2}$ are the death rates of the immature and the mature predator, respectively. $r_{1}$ denotes the rate of immature predator becoming mature predator. $\tau \geq 0$ is a constant delay due to the gestation of the predator.

The initial conditions for system (2) take the form

$$
\begin{gathered}
S(\theta)=\phi_{1}(\theta) \geq 0, \quad I(\theta)=\phi_{2}(\theta) \geq 0, \\
Y_{1}(\theta)=\varphi_{1}(\theta) \geq 0, \quad Y_{2}(\theta)=\varphi_{2}(\theta) \geq 0, \\
\theta \in[-\tau, 0), \quad \phi_{1}(0)>0, \quad \phi_{2}(0)>0, \\
\varphi_{1}(0)>0, \quad \varphi_{2}(0)>0, \\
\left(\phi_{1}(\theta), \phi_{2}(\theta), \varphi_{1}(\theta), \varphi_{2}(\theta)\right) \in C\left([-\tau, 0], R_{+0}^{4}\right),
\end{gathered}
$$

where $R_{+0}^{4}=\left\{\left(x_{1}, x_{2}, x_{3}, x_{4}\right): x_{i} \geq 0, i=1,2,3,4\right\}$.
It is well known by the fundamental theory of functional differential equations [8] that model (2) has a unique solution $\left(S(t), I(t), Y_{1}(t), Y_{2}(t)\right)$ satisfying initial conditions (3).

The organization of this paper is as follows. In the next section, we show the positivity and the boundedness of solutions of model (2) with initial conditions (3). In Section 3, we investigate the stability of the semitrivial equilibria of the model (2). In Section 4, we discuss the stability of the positive equilibrium of the model (2). Further, we study the existence of Hopf bifurcation at the positive equilibrium. A brief discussion is given in Section 5 to conclude this work.

\section{Preliminaries}

In this section, we show the positivity and the boundedness of solutions of model (2) with initial conditions (3).

Theorem 1. Solutions of model (2) with initial conditions (3) are positive for all $t \geq 0$.

Proof. Let $\left(S(t), I(t), Y_{1}(t), Y_{2}(t)\right)$ be a solution of model (2) with initial conditions (3). It follows from the first and the second equations of model (2) that

$$
\begin{aligned}
& S(t)=S(0) \exp \left\{\int_{0}^{t}\left[r-\frac{r}{K} S(s)-\left(\frac{r}{K}+\beta\right) I(s)\right] d s\right\}>0, \\
& I(t)=I(0) \exp \left\{\int_{0}^{t}\left[\beta S(s)-d-b(1-m) Y_{2}(s)\right] d s\right\}>0 .
\end{aligned}
$$

Let us consider $Y_{1}(t)$ and $Y_{2}(t)$ for $t \in[0, \tau]$. Since $\phi_{2}(\theta) \geq$ $0, \varphi_{2}(\theta) \geq 0$, for $\theta \in[-\tau, 0]$, we derive from the third equation of model (2) that

$$
\frac{d Y_{1}}{d t} \geq-\left(r_{1}+d_{1}\right) Y_{1}(t)
$$

Since $\varphi_{1}(0)>0$, a standard comparison argument shows that

$$
Y_{1}(t) \geq Y_{1}(0) e^{-\left(r_{1}+d_{1}\right) t}>0
$$

that is, $Y_{1}(t)>0$, for $t \in[0, \tau]$. For $t \in[0, \tau]$, it follows from the fourth equation of (2) that

$$
\frac{d Y_{2}}{d t} \geq-d_{2} Y_{2}(t)-a Y_{2}^{2}(t)
$$

Since $\varphi_{2}(0)>0$, a standard comparison argument shows that

$$
Y_{2}(t) \geq Y_{2}(0) \exp \left\{\int_{0}^{t}\left(-d_{2}-a Y_{2}(s)\right) d s\right\}>0 ;
$$

that is, $Y_{2}(t)>0$, for $t \in[0, \tau]$. In a similar way, we treat the intervals $[\tau, 2 \tau], \ldots,[n \tau,(n+1) \tau], n \in N$. Thus, $S(t)>$ $0, I(t)>0, Y_{1}(t)>0$, and $Y_{2}(t)>0$ for all $t \geq 0$. This completes the proof.

Theorem 2. Positive solutions of model (2) with initial conditions (3) are ultimately bounded. 
Proof. Let $\left(S(t), I(t), Y_{1}(t), Y_{2}(t)\right)$ be a positive solution of model (2) with initial conditions (3). Denote $\widehat{d}=$ $\min \left\{d, d_{1}, d_{2}\right\}$. Define

$$
W(t)=p S(t-\tau)+p I(t-\tau)+Y_{1}(t)+Y_{2}(t) .
$$

Calculating the derivative of $W(t)$ along the positive solutions of (2), it follows that

$$
\begin{aligned}
\frac{d W}{d t}= & p r S(t-\tau)-p \frac{r}{K} S^{2}(t-\tau)-p \frac{r}{K} S(t-\tau) I(t-\tau) \\
& -p d I(t-\tau)-d_{1} Y_{1}(t)-d_{2} Y_{2}(t)-a Y_{2}^{2}(t) \\
\leq & -\hat{d} W(t)+p(r+\widehat{d}) S(t-\tau)-p \frac{r}{K} S^{2}(t-\tau) \\
& -p \frac{r}{K} S(t-\tau) I(t-\tau) \\
\leq & -\widehat{d} W(t)-p \frac{r}{K}\left[S(t-\tau)-\frac{K(r+\widehat{d})}{2 r}\right]^{2} \\
& +\frac{p K(r+\widehat{d})^{2}}{4 r} \\
\leq & -\widehat{d} W(t)+\frac{p K(r+\widehat{d})^{2}}{4 r},
\end{aligned}
$$

which yields

$$
\limsup _{t \rightarrow \infty} W(t) \leq \frac{p K(r+\widehat{d})^{2}}{4 r \widehat{d}} .
$$

If we choose $M_{1}=K(r+\widehat{d})^{2} / 4 r \widehat{d}, M_{2}=p K(r+\widehat{d})^{2} / 4 r \widehat{d}$, then

$$
\begin{gathered}
\limsup _{t \rightarrow \infty} S(t) \leq M_{1}, \quad \limsup _{t \rightarrow \infty} I(t) \leq M_{1}, \\
\limsup _{t \rightarrow \infty} Y_{i}(t) \leq M_{2}, \quad(i=1,2) .
\end{gathered}
$$

This completes the proof.

\section{Boundary Equilibria and Their Stability}

In this section, we discuss the stability of the boundary equilibria of model (2).

Model (2) always has two boundary equilibria, namely, the trivial equilibrium $E_{0}(0,0,0,0)$ and the axial equilibrium $E_{K}(K, 0,0,0)$. It is easy to show that if $K \beta>d$, model (2) admits a predator-extinction equilibrium $E_{1}\left(S_{1}, I_{1}, 0,0\right)$, where

$$
S_{1}=\frac{d}{\beta}, \quad I_{1}=\frac{r(K \beta-d)}{\beta(K \beta+r)} .
$$

The characteristic equation of model (2) at the equilibrium $E_{0}(0,0,0,0)$ is of the form

$$
(\lambda-r)(\lambda+d)\left(\lambda+r_{1}+d_{1}\right)\left(\lambda+d_{2}\right)=0 .
$$

Clearly (14) has a positive real root. Accordingly, the equilibrium $E_{0}$ is unstable.

The characteristic equation of model (2) at the equilibrium $E_{K}(K, 0,0,0)$ takes the form

$$
(\lambda+r)\left(\lambda+d_{2}\right)\left(\lambda+r_{1}+d_{1}\right)[\lambda-(K \beta-d)]=0 .
$$

Hence, if $K \beta<d$, (15) has no positive real root. Accordingly, the equilibrium $E_{K}$ is locally asymptotically stable. If $K \beta>d$, (15) has a positive real root. Accordingly, the equilibrium $E_{K}$ is unstable.

Theorem 3. If $K \beta<d$, then the semitrivial equilibrium $E_{K}$ is globally stable.

Proof. Based on the above discussions, we only prove the global attractivity of the equilibrium $E_{K}$. Let

$$
\begin{aligned}
V_{K}(t)= & c_{1}\left[S(t)-K-K \ln \frac{S(t)}{K}\right]+I(t)+\frac{1}{p} Y_{1}(t) \\
& +\frac{1}{p} Y_{2}(t)+b(1-m) \int_{t-\tau}^{t} I(s) Y_{2}(s) d s,
\end{aligned}
$$

where $c_{1}=K \beta /(K \beta+r)$. Calculating the derivative of $V_{K}(t)$ along the positive solutions of model (2), it follows that

$$
\begin{aligned}
\frac{d}{d t} V_{K}(t)= & c_{1} \frac{S(t)-K}{S(t)} \dot{S}(t)+\dot{I}(t)+\frac{1}{p} \dot{Y}_{1}(t)+\frac{1}{p} \dot{Y}_{2}(t) \\
& +b(1-m) I(t) Y_{2}(t) \\
& -b(1-m) I(t-\tau) Y_{2}(t-\tau) . \\
= & -\frac{\beta r}{K \beta+r}[S(t)-K]^{2}-(d-K \beta) I(t)-\frac{1}{p} d_{1} Y_{1}(t) \\
& -\frac{1}{p} d_{2} Y_{2}(t)-\frac{a}{p} Y_{2}^{2}(t) .
\end{aligned}
$$

If $K \beta<d$, then it follows from (17) that $\dot{V}_{K}(t) \leq 0$. By Theorem 5.3.1, in [8], solutions are limited to $M$, the largest invariant subset of $\left\{\dot{V}_{K}(t)=0\right\}$. Clearly, we see from (17) that $\dot{V}_{K}(t)=0$ if and only if $S(t)=K, I(t)=0, Y_{1}(t)=$ $0, Y_{2}(t)=0$. Accordingly, the global asymptotic stability of $E_{K}$ follows from LaSalle's invariant principle. This completes the proof.

The characteristic equation of model (2) at the equilibrium $E_{1}$ is of the form

$$
\begin{aligned}
\left(\lambda^{2}+\right. & \left.\frac{r}{K} S_{1} \lambda+\frac{\beta(K \beta+r)}{K} S_{1} I_{1}\right) \\
& \times\left(\lambda^{2}+g_{1} \lambda+g_{0}+f_{0} e^{-\lambda \tau}\right)=0,
\end{aligned}
$$

where $g_{1}=r_{1}+d_{1}+d_{2}, g_{0}=d_{2}\left(r_{1}+d_{1}\right), f_{0}=-p b r_{1}(1-$ $m) I_{1}$. Clearly, the roots of equation $\lambda^{2}+(r / K) S_{1} \lambda+(\beta(K \beta+$ $r) / K) S_{1} I_{1}=0$ have negative real part. When $\tau=0$, if $\operatorname{pbrr}_{1}(1-m)(K \beta-d)<\beta d_{2}\left(r_{1}+d_{1}\right)(K \beta+r)$, then the 
roots of (18) have negative real part. Accordingly, $E_{1}$ is locally asymptotically stable. If $\operatorname{pbrr}_{1}(1-m)(K \beta-d)>\beta d_{2}\left(r_{1}+\right.$ $\left.d_{1}\right)(K \beta+r)$, then $E_{1}$ is unstable. It is easily seen that

$$
g_{1}^{2}-2 g_{0}=\left(r_{1}+d_{1}\right)^{2}+d_{2}^{2}>0, \quad g_{0}^{2}-f_{0}^{2}>0 .
$$

Hence, if $0<\operatorname{pbrr}_{1}(1-m)(K \beta-d)<\beta d_{2}\left(r_{1}+d_{1}\right)(K \beta+r)$, by Lemma B in [7], it follows that the equilibrium $E_{1}$ is locally asymptotically stable for all $\tau \geq 0$. If $\operatorname{pbrr}_{1}(1-m)(K \beta-d)>$ $\beta d_{2}\left(r_{1}+d_{1}\right)(K \beta+r)$, then $E_{1}$ is unstable for all $\tau \geq 0$.

Theorem 4. Let $K \beta>d$ hold; the predator-extinction equilibrium $E_{1}$ of model (2) is globally stable provided that

$$
\operatorname{pbrr}_{1}(1-m)(K \beta-d)<\beta d_{2}\left(r_{1}+d_{1}\right)(K \beta+r) .
$$

Proof. Based on the above discussions, we only prove the global attractivity of the equilibrium $E_{1}$. Define

$$
\begin{aligned}
V_{11}(t)= & c_{1}\left(S(t)-S_{1}-S_{1} \ln \frac{S(t)}{S_{1}}\right)+I(t)-I_{1} \\
& -I_{1} \ln \frac{I(t)}{I_{1}}+k_{1} Y_{1}(t)+k_{2} Y_{2}(t),
\end{aligned}
$$

where $c_{1}=K \beta /(K \beta+r)$ and $k_{1}=1 / p, k_{2}=\left(r_{1}+\right.$ $\left.d_{1}\right) /\left(p r_{1}\right)$. Calculating the derivative of $V_{11}(t)$ along the positive solutions of (2), it follows that

$$
\begin{aligned}
\dot{V}_{11}(t)= & c_{1} \frac{S(t)-S_{1}}{S(t)} \dot{S}(t)+\frac{I(t)-I_{1}}{I(t)} \dot{I}(t)+k_{1} \dot{Y}_{1}(t)+k_{2} \dot{Y}_{2}(t) \\
= & -\frac{\beta r}{K \beta+r}\left[S(t)-S_{1}\right]^{2}-b(1-m) I(t) Y_{2}(t) \\
& +b(1-m) I(t-\tau) Y_{2}(t-\tau) \\
& -\left[\frac{d_{2}\left(r_{1}+d_{1}\right)}{p r_{1}}-b(1-m) I_{1}\right] Y_{2}(t) \\
& -\frac{a\left(r_{1}+d_{1}\right)}{p r_{1}} Y_{2}^{2}(t) .
\end{aligned}
$$

Define

$$
V_{1}(t)=V_{11}(t)+b(1-m) \int_{t-\tau}^{t} I(s) Y_{2}(s) d s .
$$

We derive from (22) and (23) that

$$
\begin{aligned}
\dot{V}_{1}(t)= & -\frac{\beta r}{K \beta+r}\left[S(t)-S_{1}\right]^{2} \\
& -\left[\frac{d_{2}\left(r_{1}+d_{1}\right)}{p r_{1}}-b(1-m) I_{1}\right] Y_{2}(t) \\
& -\frac{a\left(r_{1}+d_{1}\right)}{p r_{1}} Y_{2}^{2}(t) .
\end{aligned}
$$

If $0<\operatorname{pbrr}_{1}(1-m)(K \beta-d)<\beta d_{2}\left(r_{1}+d_{1}\right)(K \beta+r)$, it then follows from $(24)$ that $\dot{V}_{1}(t) \leq 0$. By Theorem 5.3.1, in
[8], solutions are limited to $M$, the largest invariant subset of $\left\{\dot{V}_{1}(t)=0\right\}$. Clearly, we see from $(24)$ that $\dot{V}_{1}(t)=0$, if and only if $S(t)=S_{1}, Y_{2}(t)=0$. It follows from the first and fourth equations of (2) that $0=\dot{S}(t)=r-(r / K) S_{1}-((K \beta+$ $r) / K) I(t), 0=\dot{Y}_{2}(t)=r_{1} Y_{1}(t)$, which yields $I(t)=I_{1}, Y_{1}(t)=$ 0 . Using LaSalle's invariant principle, the global asymptotic stability of $E_{1}$ follows. This completes the proof.

\section{Stability of Positive Equilibrium}

In this section, we are concerned with the stability of the positive equilibrium $E^{*}$ and the existence of Hopf bifurcations at the positive equilibrium $E^{*}$ of model (2).

If the following holds,

(H1) $\operatorname{pbrr}_{1}(1-m)(K \beta-d)>\beta d_{2}\left(r_{1}+d_{1}\right)(K \beta+r)$, then model (2) has a unique positive equilibrium $E^{*}\left(S^{*}, I^{*}, Y_{1}^{*}, Y_{2}^{*}\right)$, where

$$
\begin{aligned}
& S^{*}=\left(K \operatorname{Krr}_{1} b^{2}(1-m)^{2}+a d\left(r_{1}+d_{1}\right)(K \beta+r)\right. \\
&\left.-b(1-m) d_{2}\left(r_{1}+d_{1}\right)(K \beta+r)\right) \\
& \times\left(\operatorname{prr}_{1} b^{2}(1-m)^{2}+\beta a\left(r_{1}+d_{1}\right)(K \beta+r)\right)^{-1}, \\
& I^{*}=\frac{r\left(K-S^{*}\right)}{K \beta+r}, \quad Y_{1}^{*}=\frac{d_{2}+a Y_{2}^{*}}{r_{1}} Y_{2}^{*}, \\
& Y_{2}^{*}=\frac{\beta S^{*}-d}{b(1-m)} .
\end{aligned}
$$

The characteristic equation of model (2) at the equilibrium $E^{*}$ takes the form

$$
\lambda^{4}+p_{3} \lambda^{3}+p_{2} \lambda^{2}+p_{1} \lambda+p_{0}+\left(q_{2} \lambda^{2}+q_{1} \lambda+q_{0}\right) e^{-\lambda \tau}=0,
$$

where

$$
\begin{aligned}
p_{3}= & r_{1}+d_{1}+d_{2}+2 a Y_{2}^{*}+\frac{r}{K} S^{*} \\
p_{2}= & \left(r_{1}+d_{1}\right)\left(d_{2}+2 a Y_{2}^{*}\right)+\frac{r}{K} S^{*}\left(r_{1}+d_{1}+d_{2}+2 a Y_{2}^{*}\right) \\
& +\frac{K \beta+r}{K} \beta S^{*} I^{*} \\
p_{1}= & \frac{K \beta+r}{K} \beta S^{*} I^{*}\left(r_{1}+d_{1}+d_{2}+2 a Y_{2}^{*}\right) \\
& +\frac{r}{K} S^{*}\left(r_{1}+d_{1}\right)\left(d_{2}+2 a Y_{2}^{*}\right), \\
p_{0}= & \frac{K \beta+r}{K} \beta S^{*} I^{*}\left(r_{1}+d_{1}\right)\left(d_{2}+2 a Y_{2}^{*}\right), \\
q_{2}= & -p b r_{1}(1-m) I^{*}, \\
q_{1}= & p b r_{1}(1-m) I^{*}\left[b(1-m) Y_{2}^{*}-\frac{r}{K} S^{*}\right], \\
q_{0}= & p b r_{1}(1-m) S^{*} I^{*}\left[b(1-m) \frac{r}{K} Y_{2}^{*}-\frac{K \beta+r}{K} \beta I^{*}\right]
\end{aligned}
$$


It is easy to show that

$$
p_{3}>0, \quad p_{0}+q_{0}>0, \quad p_{1}+q_{1}>0, \quad p_{2}+q_{2}>0 .
$$

When $\tau=0$, (26) becomes

$$
\lambda^{4}+p_{3} \lambda^{3}+\left(p_{2}+q_{2}\right) \lambda^{2}+\left(p_{1}+q_{1}\right) \lambda+p_{0}+q_{0}=0 .
$$

If the following holds,

(H2) $\left(p_{1}+q_{1}\right)\left[p_{3}\left(p_{2}+q_{2}\right)-\left(p_{0}+q_{0}\right)\right]>p_{3}^{2}\left(p_{0}+q_{0}\right)$, then by the Routh-Hurwitz theorem, when $\tau=0$, the coexistence equilibrium $E^{*}$ of model (2) is locally asymptotically stable and $E^{*}$ is unstable if $\left(p_{1}+\right.$ $\left.q_{1}\right)\left[p_{3}\left(p_{2}+q_{2}\right)-\left(p_{0}+q_{0}\right)\right]<p_{3}^{2}\left(p_{0}+q_{0}\right)$.

If $i \omega(\omega>0)$ is a solution of (26), separating real and imaginary parts, we have

$$
\begin{gathered}
\left(q_{2} \omega^{2}-q_{0}\right) \sin \omega \tau+q_{1} \omega \cos \omega \tau=p_{3} \omega^{3}-p_{1} \omega, \\
\left(q_{2} \omega^{2}-q_{0}\right) \cos \omega \tau-q_{1} \omega \sin \omega \tau=\omega^{4}-p_{2} \omega^{2}+p_{0} .
\end{gathered}
$$

Squaring and adding the two equations of (30), it follows that

$$
\omega^{8}+h_{3} \omega^{6}+h_{2} \omega^{4}+h_{1} \omega^{2}+h_{0}=0
$$

where

$$
\begin{aligned}
& h_{3}=p_{3}^{2}-2 p_{2}, \quad h_{2}=p_{2}^{2}+2 p_{0}-2 p_{1} p_{3}-q_{2}^{2}, \\
& h_{1}=p_{1}^{2}-2 p_{0} p_{2}+2 q_{0} q_{2}-q_{1}^{2}, \quad h_{0}=p_{0}^{2}-q_{0}^{2} .
\end{aligned}
$$

Assume that the following holds:

$$
\text { (H3) } h_{3}>0, h_{2}>0, h_{1}>0 \text {. }
$$

If $h_{0}>0$, by the general theory on characteristic equations of delay differential equations from [9] (Theorem 4.1), $E^{*}$ remains stable for all $\tau>0$. If $h_{0}<0$, then (31) has a unique positive root $\omega_{0}$; that is, (26) admits a pair of purely imaginary roots of the form $\pm i \omega_{0}$. From (30), we see that

$$
\begin{aligned}
\tau_{n}= & \frac{2 n \pi}{\omega_{0}}+\frac{1}{\omega_{0}} \arccos \left(\left(q_{2} \omega_{0}^{2}-q_{0}\right)\left(\omega_{0}^{4}-p_{2} \omega_{0}^{2}+p_{0}\right)\right. \\
& \left.+q_{1} \omega_{0}\left(p_{3} \omega_{0}^{3}-p_{1} \omega_{0}\right)\right) \\
& \times\left(\left(q_{1} \omega_{0}\right)^{2}+\left(q_{2} \omega_{0}^{2}-q_{0}\right)^{2}\right)^{-1}, \quad n=0,1,2, \ldots
\end{aligned}
$$

By Theorem 3.4.1, in [9], we see that $E^{*}$ remains stable for $\tau<\tau_{0}$.

In the following, we claim that

$$
\left.\frac{d(\operatorname{Re}(\lambda))}{d \tau}\right|_{\tau=\tau_{0}}>0 .
$$

This will show that there exists at least one eigenvalue with a positive real part for $\tau>\tau_{0}$. Moreover, the conditions for the existence of a Hopf bifurcation (Theorem 2.9.1 in [9]) are then satisfied yielding a periodic solution. To this end, differentiating equation (26) with respect to $\tau$, it follows that

$$
\begin{aligned}
\left(\frac{d \lambda}{d \tau}\right)^{-1}= & \frac{4 \lambda^{3}+3 p_{3} \lambda^{2}+2 p_{2} \lambda+p_{1}}{-\lambda\left(\lambda^{4}+p_{3} \lambda^{3}+p_{2} \lambda^{2}+p_{1} \lambda+p_{0}\right)} \\
& +\frac{2 q_{2} \lambda+q_{1}}{\lambda\left(q_{2} \lambda^{2}+q_{1} \lambda+q_{0}\right)}-\frac{\tau}{\lambda} .
\end{aligned}
$$

Hence, a direct calculation shows that

$$
\begin{aligned}
\operatorname{sgn}\left\{\frac{d(\operatorname{Re} \lambda)}{d \tau}\right\}_{\lambda=i \omega_{0}} & \left.\operatorname{Re}\left(\frac{d \lambda}{d \tau}\right)^{-1}\right\}_{\lambda=i \omega_{0}} \\
=\operatorname{sgn}\{\operatorname{sgn}\{ & \left(\left(3 p_{3} \omega_{0}^{2}-p_{1}\right)\left(p_{3} \omega_{0}^{2}-p_{1}\right)+2\left(2 \omega_{0}^{2}-p_{2}\right)\right. \\
& \left.\times\left(\omega_{0}^{4}-p_{2} \omega_{0}^{2}+p_{0}\right)\right) \\
\times & \left(\omega_{0}^{2}\left(p_{1}-p_{3} \omega_{0}^{2}\right)^{2}+\left(\omega_{0}^{4}-p_{2} \omega_{0}^{2}+p_{0}\right)^{2}\right)^{-1} \\
+ & \left(-q_{1}^{2}+2 q_{2}\left(q_{0}-q_{2} \omega_{0}^{2}\right)\right) \\
\times & \left.\left(\left(q_{1} \omega_{0}\right)^{2}+\left(q_{2} \omega_{0}^{2}-q_{0}\right)^{2}\right)^{-1}\right\} .
\end{aligned}
$$

We derive from (30) that

$$
\begin{gathered}
\omega_{0}^{2}\left(p_{1}-p_{3} \omega_{0}^{2}\right)^{2}+\left(\omega_{0}^{4}-p_{2} \omega_{0}^{2}+p_{0}\right)^{2} \\
=\left(q_{1} \omega_{0}\right)^{2}+\left(q_{2} \omega_{0}^{2}-q_{0}\right)^{2} .
\end{gathered}
$$

Hence, it follows that

$$
\begin{aligned}
\operatorname{sgn}\left\{\frac{d(\operatorname{Re} \lambda)}{d \tau}\right\}_{\lambda=i \omega_{0}} \\
\quad=\operatorname{sgn}\left\{\frac{4 \omega_{0}^{6}+3 h_{3} \omega_{0}^{4}+2 h_{2} \omega_{0}^{2}+h_{1}}{\left(q_{1} \omega_{0}\right)^{2}+\left(q_{2} \omega_{0}^{2}-q_{0}\right)^{2}}\right\}>0 .
\end{aligned}
$$

Therefore, if (H3) holds, then the transversal condition holds and a Hopf bifurcation occurs at $\omega=\omega_{0}, \tau=\tau_{0}$.

In conclusion, we have the following results.

Theorem 5. For model (2), let (H1) hold, and we have the following.

(i) If (H2) and (H3) hold, $h_{0}>0$, then the positive equilibrium $E^{*}$ is locally asymptotically stable for all $\tau \geq 0$.

(ii) If (H2) and (H3) hold, $h_{0}<0$, then there exists a positive number $\tau_{0}$, such that the positive equilibrium $E^{*}$ is locally asymptotically stable if $0 \leq \tau<\tau_{0}$ and is unstable if $\tau>\tau_{0}$. Further, model (2) undergoes a Hopf bifurcation at $E^{*}$ when $\tau=\tau_{0}$. 
(iii) If $\left(p_{1}+q_{1}\right)\left[p_{3}\left(p_{2}+q_{2}\right)-\left(p_{1}+q_{1}\right)\right]<p_{3}^{2}\left(p_{0}+q_{0}\right)$, then the positive equilibrium $E^{*}$ is unstable for all $\tau \geq 0$.

Now, we are concerned with the global attractiveness of the positive equilibrium $E^{*}$.

Theorem 6. Let (H1) hold, and then the positive equilibrium $E^{*}\left(S^{*}, I^{*}, Y_{1}^{*}, Y_{2}^{*}\right)$ of model (2) is globally attractive provided that

$$
a \beta\left(r_{1}+d_{1}\right)(K \beta+r) \neq \operatorname{prr}_{1} b^{2}(1-m)^{2} .
$$

Proof. Let $\left(S(t), I(t), Y_{1}(t), Y_{2}(t)\right)$ be any positive solution of model (2) with initial conditions (3). Let

$$
\begin{gathered}
M_{S}=\limsup _{t \rightarrow+\infty} S(t), \quad m_{S}=\liminf _{t \rightarrow+\infty} S(t), \\
M_{I}=\lim _{t \rightarrow+\infty} \sup _{t} I(t), \\
m_{I}=\lim _{t \rightarrow+\infty} \inf _{t}(t), \\
M_{Y_{i}}=\lim _{t \rightarrow+\infty} \sup _{i} Y_{i}(t), \quad m_{Y_{i}}=\liminf _{t \rightarrow+\infty} Y_{i}(t), \quad(i=1,2) .
\end{gathered}
$$

We now claim that $M_{S}=m_{S}=S^{*}, M_{I}=m_{I}=I^{*}, M_{Y_{i}}=$ $m_{Y_{i}}=Y_{i}^{*}(i=1,2)$. The technique of proof is to use an iteration method.

We derive from the first and the second equations of model (2) that

$$
\frac{d S}{d t}=r S\left(1-\frac{S+I}{K}\right)-\beta S I, \quad \frac{d I}{d t} \leq \beta S I-d I .
$$

Consider the following auxiliary equations:

$$
\begin{aligned}
& \frac{d x_{1}}{d t}=r x_{1}\left(1-\frac{x_{1}+x_{2}}{K}\right)-\beta x_{1} x_{2}, \\
& \frac{d x_{2}}{d t}=\beta x_{1} x_{2}-d x_{2} .
\end{aligned}
$$

If $K \beta>d$, then, by Theorem 3.1 in [2], it follows from (42) that

$$
\lim _{t \rightarrow+\infty} x_{1}(t)=\frac{d}{\beta}, \quad \lim _{t \rightarrow+\infty} x_{2}(t)=\frac{r(K \beta-d)}{\beta(K \beta+r)} .
$$

By comparison, we obtain that

$$
\begin{gathered}
M_{S}=\limsup _{t \rightarrow+\infty} S(t) \leq \frac{d}{\beta}:=M_{1}^{S}, \\
M_{I}=\lim _{t \rightarrow+\infty} \sup _{t \rightarrow+\infty}(t) \leq \frac{r(K \beta-d)}{\beta(K \beta+r)}:=M_{1}^{I} .
\end{gathered}
$$

Hence, for $\varepsilon>0$, sufficiently small, there is a $T_{1}>0$ such that if $t>T_{1}$, then $I(t) \leq M_{1}^{I}+\varepsilon$. We therefore derive from the third and the fourth equations of model (2) that, for $t>T_{1}+\tau$,

$$
\begin{aligned}
& \frac{d Y_{1}}{d t} \leq p b(1-m)\left(M_{1}^{I}+\varepsilon\right) Y_{2}(t-\tau)-\left(r_{1}+d_{1}\right) Y_{1}(t), \\
& \frac{d Y_{2}}{d t}=r_{1} Y_{1}(t)-d_{2} Y_{2}(t)-a Y_{2}^{2}(t) .
\end{aligned}
$$

Consider the following auxiliary equations:

$$
\begin{gathered}
\frac{d z_{1}}{d t}=p b(1-m)\left(M_{1}^{I}+\varepsilon\right) z_{2}(t-\tau)-\left(r_{1}+d_{1}\right) z_{1}(t), \\
\frac{d z_{2}}{d t}=r_{1} z_{1}(t)-d_{2} z_{2}(t)-a z_{2}^{2}(t) .
\end{gathered}
$$

If (H1) holds, then, by Lemma 2.4 in [10], it follows from (46) that

$$
\begin{aligned}
\lim _{t \rightarrow+\infty} z_{1}(t)= & \left(p b(1-m)\left(M_{1}^{I}+\varepsilon\right)\right. \\
& \left.\times\left[p b r_{1}(1-m)\left(M_{1}^{I}+\varepsilon\right)-d_{2}\left(r_{1}+d_{1}\right)\right]\right) \\
& \times\left(a\left(r_{1}+d_{1}\right)^{2}\right)^{-1}, \\
\lim _{t \rightarrow+\infty} z_{2}(t) & =\frac{p b r_{1}(1-m)\left(M_{1}^{I}+\varepsilon\right)-d_{2}\left(r_{1}+d_{1}\right)}{a\left(r_{1}+d_{1}\right)} .
\end{aligned}
$$

By comparison, for $\varepsilon>0$, sufficiently small, we obtain that

$$
\begin{aligned}
M_{Y_{1}} & =\limsup _{t \rightarrow+\infty} Y_{1}(t) \\
& \leq \frac{p b(1-m) M_{1}^{I}\left[p b r_{1}(1-m) M_{1}^{I}-d_{2}\left(r_{1}+d_{1}\right)\right]}{a\left(r_{1}+d_{1}\right)^{2}} \\
& :=M_{1}^{Y_{1}}, \\
M_{Y_{2}} & =\limsup _{t \rightarrow+\infty} Y_{2}(t) \\
& =\frac{p b r_{1}(1-m) M_{1}^{I}-d_{2}\left(r_{1}+d_{1}\right)}{a\left(r_{1}+d_{1}\right)} \\
& :=M_{1}^{Y_{2}} . \quad
\end{aligned}
$$

Hence, for $\varepsilon>0$, sufficiently small, there is a $T_{2} \geq T_{1}+\tau$ such that if $t>T_{2}$, then $Y_{2}(t) \leq M_{1}^{Y_{2}}+\varepsilon$.

For $\varepsilon>0$, sufficiently small, we derive from the first and the second equations of model (2) that, for $t>T_{2}$,

$$
\begin{gathered}
\frac{d S}{d t}=r S\left(1-\frac{S+I}{K}\right)-\beta S I, \\
\frac{d I}{d t} \geq \beta S I-d I-b(1-m)\left(M_{1}^{Y_{2}}+\varepsilon\right) I .
\end{gathered}
$$

Consider the following auxiliary equations:

$$
\begin{aligned}
& \frac{d x_{1}}{d t}=r x_{1}\left(1-\frac{x_{1}+x_{2}}{K}\right)-\beta x_{1} x_{2}, \\
& \frac{d x_{2}}{d t}=\beta x_{1} x_{2}-d x_{2}-b(1-m)\left(M_{1}^{Y_{2}}+\varepsilon\right) x_{2} .
\end{aligned}
$$


If (H1) holds, then, by Theorem 3.1 in [2], it follows from (50) that

$$
\begin{aligned}
& \lim _{t \rightarrow+\infty} x_{1}(t)=\frac{d+b(1-m)\left(M_{1}^{Y_{2}}+\varepsilon\right)}{\beta}, \\
& \lim _{t \rightarrow+\infty} x_{2}(t)=\frac{r}{K \beta+r}\left[K-\frac{d+b(1-m)\left(M_{1}^{Y_{2}}+\varepsilon\right)}{\beta}\right] .
\end{aligned}
$$

By comparison, for $\varepsilon>0$, sufficiently small, we conclude that

$$
\begin{aligned}
& m_{S}=\liminf _{t \rightarrow+\infty} S(t) \geq \frac{d+b(1-m) M_{1}^{Y_{2}}}{\beta}:=N_{1}^{S}, \\
& m_{I}=\liminf _{t \rightarrow+\infty} \geq \frac{r}{K \beta+r}\left(K-\frac{d+b(1-m) M_{1}^{Y_{2}}}{\beta}\right):=N_{1}^{I} .
\end{aligned}
$$

Hence, for $\varepsilon>0$, sufficiently small, there is a $T_{3} \geq T_{2}$ such that if $t>T_{3}$, then $I(t) \geq N_{1}^{I}-\varepsilon$. For $\varepsilon>0$, sufficiently small, we derive from the third and the fourth equations of model (2) that for $t>T_{3}+\tau$

$$
\begin{aligned}
& \frac{d Y_{1}}{d t} \geq p b(1-m)\left(N_{1}^{I}-\varepsilon\right) Y_{2}(t-\tau)-\left(d_{1}+r_{1}\right) Y_{1}(t), \\
& \frac{d Y_{2}}{d t}=r_{1} Y_{1}(t)-d_{2} Y_{2}(t)-a Y_{2}^{2}(t) .
\end{aligned}
$$

Consider the following auxiliary equations:

$$
\begin{aligned}
& \frac{d z_{1}}{d t}=p b(1-m)\left(N_{1}^{I}-\varepsilon\right) z_{2}(t-\tau)-\left(r_{1}+d_{1}\right) z_{1}(t) \\
& \frac{d z_{2}}{d t}=r_{1} z_{1}(t)-d_{2} z_{2}(t)-a z_{2}^{2}(t)
\end{aligned}
$$

Since (H1) holds, by Lemma 2.4 of [10], it follows from (54) that

$$
\begin{aligned}
\lim _{t \rightarrow+\infty} z_{1}(t)=( & p b(1-m)\left(N_{1}^{I}-\varepsilon\right) \\
\times & {\left.\left[p b r_{1}(1-m)\left(N_{1}^{I}-\varepsilon\right)-d_{2}\left(r_{1}+d_{1}\right)\right]\right) } \\
& \times\left(a\left(r_{1}+d_{1}\right)^{2}\right)^{-1}, \\
\lim _{t \rightarrow+\infty} z_{2}(t)= & \frac{p b r_{1}(1-m)\left(N_{1}^{I}-\varepsilon\right)-d_{2}\left(r_{1}+d_{1}\right)}{a\left(r_{1}+d_{1}\right)} .
\end{aligned}
$$

By comparison, for $\varepsilon>0$, sufficiently small, we obtain that

$$
\begin{aligned}
m_{Y_{1}} & =\liminf _{t \rightarrow+\infty} Y_{1}(t) \\
& \geq \frac{p b(1-m) N_{1}^{I}\left[p b r_{1}(1-m) N_{1}^{I}-d_{2}\left(r_{1}+d_{1}\right)\right]}{a\left(r_{1}+d_{1}\right)^{2}} \\
& :=N_{1}^{Y_{1}}, \\
m_{Y_{2}} & =\liminf _{t \rightarrow+\infty} Y_{2}(t) \\
& \geq \frac{p b r_{1}(1-m) N_{1}^{I}-d_{2}\left(r_{1}+d_{1}\right)}{a\left(r_{1}+d_{1}\right)} \\
& :=N_{1}^{Y_{2}} .
\end{aligned}
$$

Hence, for $\varepsilon>0$, sufficiently small, there is a $T_{4} \geq T_{3}+\tau$, such that if $t>T_{4}, Y_{2}(t) \geq N_{1}^{Y_{2}}-\varepsilon$.

For $\varepsilon>0$, sufficiently small, we derive from the first and the second equations of model (2) that, for $t>T_{4}$,

$$
\begin{gathered}
\frac{d S}{d t}=r S\left(1-\frac{S+I}{K}\right)-\beta S I, \\
\frac{d I}{d t} \leq \beta S I-d I-b(1-m)\left(N_{1}^{Y_{2}}-\varepsilon\right) I .
\end{gathered}
$$

Consider the following auxiliary equations:

$$
\begin{aligned}
& \frac{d x_{1}}{d t}=r x_{1}\left(1-\frac{x_{1}+x_{2}}{K}\right)-\beta x_{1} x_{2}, \\
& \frac{d x_{2}}{d t}=\beta x_{1} x_{2}-d x_{2}-b(1-m)\left(N_{1}^{Y_{2}}-\varepsilon\right) x_{2} .
\end{aligned}
$$

If (H1) holds, then, by Theorem 3.1 in [2], it follows from (58) that

$$
\begin{gathered}
\lim _{t \rightarrow+\infty} x_{1}(t)=\frac{d+b(1-m)\left(N_{1}^{Y_{2}}-\varepsilon\right)}{\beta}, \\
\lim _{t \rightarrow+\infty} x_{2}(t)=\frac{r}{K \beta+r}\left[K-\frac{d+b(1-m)\left(N_{1}^{Y_{2}}-\varepsilon\right)}{\beta}\right] .
\end{gathered}
$$

By comparison, for $\varepsilon>0$, sufficiently small, we obtain that

$$
\begin{aligned}
& M_{S}=\limsup _{t \rightarrow+\infty} S(t) \leq \frac{d+b(1-m) N_{1}^{Y_{2}}}{\beta}:=M_{2}^{S}, \\
& M_{I}=\limsup _{t \rightarrow+\infty} \leq \frac{r}{K \beta+r}\left(K-\frac{d+b(1-m) N_{1}^{Y_{2}}}{\beta}\right):=M_{2}^{I} .
\end{aligned}
$$

Therefore, for $\varepsilon>0$, sufficiently small, there is a $T_{5} \geq T_{4}$ such that if $t>T_{5}, I(t) \leq M_{2}^{I}+\varepsilon$. 
For $\varepsilon>0$, sufficiently small, we derive from the third and the fourth equations of model (2) that, for $t>T_{5}+\tau$,

$$
\begin{aligned}
& \frac{d Y_{1}}{d t} \leq p b(1-m)\left(M_{2}^{I}+\varepsilon\right) Y_{2}(t-\tau)-\left(d_{1}+r_{1}\right) Y_{1}(t), \\
& \frac{d Y_{2}}{d t}=r_{1} Y_{1}(t)-d_{2} Y_{2}(t)-a Y_{2}^{2}(t) .
\end{aligned}
$$

Consider the following auxiliary equations:

$$
\begin{aligned}
& \frac{d z_{1}}{d t}=p b(1-m)\left(M_{2}^{I}+\varepsilon\right) z_{2}(t-\tau)-\left(r_{1}+d_{1}\right) z_{1}(t), \\
& \frac{d z_{2}}{d t}=r_{1} z_{1}(t)-d_{2} z_{2}(t)-a z_{2}^{2}(t) .
\end{aligned}
$$

Since (H1) holds, by Lemma 2.4 of [10], it follows from (62) that

$$
\begin{aligned}
\lim _{t \rightarrow+\infty} z_{1}(t)=( & p b(1-m)\left(M_{2}^{I}+\varepsilon\right) \\
& \left.\times\left[p b r_{1}(1-m)\left(M_{2}^{I}+\varepsilon\right)-d_{2}\left(r_{1}+d_{1}\right)\right]\right) \\
& \times\left(a\left(r_{1}+d_{1}\right)^{2}\right)^{-1}, \\
\lim _{t \rightarrow+\infty} z_{2}(t)= & \frac{p b r_{1}(1-m)\left(M_{2}^{I}+\varepsilon\right)-d_{2}\left(r_{1}+d_{1}\right)}{a\left(r_{1}+d_{1}\right)} .
\end{aligned}
$$

By comparison, for $\varepsilon>0$, sufficiently small, we conclude that

$$
\begin{aligned}
M_{Y_{1}} & =\limsup _{t \rightarrow+\infty} Y_{1}(t) \\
& \geq \frac{p b(1-m) M_{2}^{I}\left[p b r_{1}(1-m) M_{2}^{I}-d_{2}\left(r_{1}+d_{1}\right)\right]}{a\left(r_{1}+d_{1}\right)^{2}} \\
& :=M_{2}^{Y_{1}}, \\
M_{Y_{2}} & =\limsup _{t \rightarrow+\infty} Y_{2}(t) \\
& \geq \frac{p b r_{1}(1-m) M_{2}^{I}-d_{2}\left(r_{1}+d_{1}\right)}{a\left(r_{1}+d_{1}\right)} \\
& :=M_{2}^{Y_{2}} .
\end{aligned}
$$

Therefore, for $\varepsilon>0$, sufficiently small, there is a $T_{6} \geq T_{5}+\tau$ such that if $t>T_{6}, y_{2}(t) \leq M_{2}^{Y_{2}}+\varepsilon$.

For $\varepsilon>0$, sufficiently small, it follows from the first and the second equations of model (2) that for $t>T_{6}$

$$
\begin{aligned}
& \frac{d S}{d t}=r S\left(1-\frac{S+I}{K}\right)-\beta S I, \\
& \frac{d I}{d t} \geq \beta S I-d I-b(1-m)\left(M_{2}^{Y_{2}}+\varepsilon\right) I(t) .
\end{aligned}
$$

Consider the following auxiliary equations:

$$
\begin{aligned}
& \frac{d x_{1}}{d t}=r x_{1}\left(1-\frac{x_{1}+x_{2}}{K}\right)-\beta x_{1} x_{2}, \\
& \frac{d x_{2}}{d t}=\beta x_{1} x_{2}-d x_{2}-b(1-m)\left(M_{2}^{Y_{2}}+\varepsilon\right) x_{2} .
\end{aligned}
$$

If (H1) holds, then, by Theorem 3.1 in [2], it follows from (66) that

$$
\begin{aligned}
& \lim _{t \rightarrow+\infty} x_{1}(t)=\frac{d+b(1-m)\left(M_{2}^{Y_{2}}+\varepsilon\right)}{\beta}, \\
& \lim _{t \rightarrow+\infty} x_{2}(t)=\frac{r}{K \beta+r}\left[K-\frac{d+b(1-m)\left(M_{2}^{Y_{2}}+\varepsilon\right)}{\beta}\right] .
\end{aligned}
$$

By comparison, for $\varepsilon>0$, sufficiently small, we obtain that

$$
\begin{aligned}
& m_{S}=\liminf _{t \rightarrow+\infty} S(t) \leq \frac{d+b(1-m) M_{2}^{Y_{2}}}{\beta}:=N_{2}^{S}, \\
& m_{I}=\liminf _{t \rightarrow+\infty} \leq \frac{r}{K \beta+r}\left(K-\frac{d+b(1-m) M_{2}^{Y_{2}}}{\beta}\right):=N_{2}^{I} .
\end{aligned}
$$

Hence, for $\varepsilon>0$, sufficiently small, there is a $T_{7} \geq T_{6}$ such that if $t>T_{7}, I(t) \geq N_{2}^{I}-\varepsilon$. We therefore obtain from the third and the fourth equations of model (2) that for $t>T_{7}+\tau$

$$
\begin{aligned}
& \frac{d Y_{1}}{d t} \geq p b(1-m)\left(N_{2}^{I}-\varepsilon\right) Y_{2}(t-\tau)-\left(d_{1}+r_{1}\right) Y_{1}(t), \\
& \frac{d Y_{2}}{d t}=r_{1} Y_{1}(t)-d_{2} Y_{2}(t)-a Y_{2}^{2}(t) .
\end{aligned}
$$

Consider the following auxiliary equations:

$$
\begin{aligned}
& \frac{d z_{1}}{d t}=p b(1-m)\left(N_{2}^{I}-\varepsilon\right) z_{2}(t-\tau)-\left(r_{1}+d_{1}\right) z_{1}(t), \\
& \frac{d z_{2}}{d t}=r_{1} z_{1}(t)-d_{2} z_{2}(t)-a z_{2}^{2}(t) .
\end{aligned}
$$

Since (H1) holds, by Lemma 2.4 of [10], it follows from (70) that

$$
\begin{aligned}
\lim _{t \rightarrow+\infty} z_{1}(t)=( & p b(1-m)\left(N_{2}^{I}-\varepsilon\right) \\
& \left.\times\left[p b r_{1}(1-m)\left(N_{2}^{I}-\varepsilon\right)-d_{2}\left(r_{1}+d_{1}\right)\right]\right) \\
& \times\left(a\left(r_{1}+d_{1}\right)^{2}\right)^{-1}, \\
\lim _{t \rightarrow+\infty} z_{2}(t)= & \frac{p b r_{1}(1-m)\left(N_{2}^{I}-\varepsilon\right)-d_{2}\left(r_{1}+d_{1}\right)}{a\left(r_{1}+d_{1}\right)} .
\end{aligned}
$$


By comparison, for $\varepsilon>0$, sufficiently small, we obtain that

$$
\begin{aligned}
m_{Y_{1}} & =\liminf _{t \rightarrow+\infty} Y_{1}(t) \\
& \geq \frac{p b(1-m) N_{2}^{I}\left[p b r_{1}(1-m) N_{2}^{I}-d_{2}\left(r_{1}+d_{1}\right)\right]}{a\left(r_{1}+d_{1}\right)^{2}} \\
& :=N_{2}^{Y_{1}}, \\
m_{Y_{2}} & =\liminf _{t \rightarrow+\infty} Y_{2}(t) \\
& \geq \frac{p b r_{1}(1-m) N_{2}^{I}-d_{2}\left(r_{1}+d_{1}\right)}{a\left(r_{1}+d_{1}\right)} \\
& :=N_{2}^{Y_{2}} .
\end{aligned}
$$

Continuing this process, we derive eight sequences $M_{k}^{S}, M_{k}^{I}, M_{k}^{Y_{1}}, M_{k}^{Y_{2}}, N_{k}^{S}, N_{k}^{I}, N_{k}^{Y_{1}}, N_{k}^{Y_{2}}(k=1,2, \ldots)$ such that, for $k \geq 2$,

$$
\begin{aligned}
M_{k}^{S} & =\frac{d+b(1-m) N_{k-1}^{Y_{2}}}{\beta}, \\
M_{k}^{I} & =\frac{r}{r+K \beta}\left(K-M_{k}^{S}\right), \\
M_{k}^{Y_{1}} & =\frac{p b(1-m) M_{k}^{Y_{2}} M_{k}^{I}}{r_{1}+d_{1}}, \\
M_{k}^{Y_{2}} & =\frac{p b r_{1}(1-m) M_{k}^{I}-d_{2}\left(r_{1}+d_{1}\right)}{a\left(r_{1}+d_{1}\right)}, \\
N_{k}^{S} & =\frac{d+b(1-m) M_{k}^{Y_{2}}}{\beta}, \\
N_{k}^{I} & =\frac{r}{r+K \beta}\left(K-N_{k}^{S}\right), \\
N_{k}^{Y_{1}} & =\frac{p b(1-m) N_{k}^{Y_{2}} N_{k}^{I}}{r_{1}+d_{1}}, \\
N_{k}^{Y_{2}} & =\frac{p b r_{1}(1-m) N_{k}^{I}-d_{2}\left(r_{1}+d_{1}\right)}{a\left(r_{1}+d_{1}\right)} .
\end{aligned}
$$

It is readily seen that

$$
\begin{gathered}
N_{k}^{S} \leq m_{S} \leq M_{S} \leq M_{k}^{S}, \quad N_{k}^{I} \leq m_{I} \leq M_{I} \leq M_{k}^{I}, \\
N_{k}^{Y_{i}} \leq m_{Y_{i}} \leq M_{Y_{i}} \leq M_{k}^{Y_{i}} \quad(i=1,2) .
\end{gathered}
$$

Note that the sequences $M_{k}^{S}, M_{k}^{I}, M_{k}^{Y_{1}}, M_{k}^{Y_{2}}$ are nonincreasing and the sequences $N_{k}^{S}, N_{k}^{I}, N_{k}^{Y_{1}}, N_{k}^{Y_{2}}$ are nondecreasing. Hence, the limit of each sequence in $M_{k}^{S}, M_{k}^{I}, M_{k}^{Y_{1}}, M_{k}^{Y_{2}}, N_{k}^{S}, N_{k}^{I}, N_{k}^{Y_{1}}, N_{k}^{Y_{2}}$ exists. Denote

$$
\begin{array}{ll}
\lim _{k \rightarrow+\infty} M_{k}^{S}=\bar{S}, & \lim _{k \rightarrow+\infty} M_{k}^{I}=\bar{I}, \\
\lim _{k \rightarrow+\infty} M_{k}^{Y_{i}}=\bar{Y}_{i}, & (i=1,2), \\
\lim _{k \rightarrow+\infty} N_{k}^{S}=\underline{S}, & \lim _{k \rightarrow+\infty} N_{k}^{I}=\underline{I}, \\
\lim _{k \rightarrow+\infty} N_{k}^{Y_{i}}=\underline{Y}_{i}, \quad(i=1,2) .
\end{array}
$$

From (73), we can obtain

$$
\begin{aligned}
\bar{S} & =\frac{1}{\beta}\left[d+b(1-m) \underline{Y}_{2}\right], \\
\bar{I} & =\frac{r}{r+K \beta}(K-\bar{S}), \\
\bar{Y}_{1} & =\frac{p b(1-m) \overline{Y_{2} I}}{r_{1}+d_{1}}, \\
\bar{Y}_{2} & =\frac{p b r_{1}(1-m) \bar{I}-d_{2}\left(r_{1}+d_{1}\right)}{a\left(r_{1}+d_{1}\right)}, \\
\underline{S}^{\prime} & =\frac{1}{\beta}\left[d+b(1-m) \bar{Y}_{2}\right], \\
\underline{I} & =\frac{r}{r+K \beta}(K-\underline{S}), \\
\underline{Y}_{1} & =\frac{p b(1-m) \underline{Y_{2}} \underline{I}}{r_{1}+d_{1}}, \\
\underline{Y}_{2} & =\frac{p b r_{1}(1-m) \underline{I}-d_{2}\left(r_{1}+d_{1}\right)}{a\left(r_{1}+d_{1}\right)} .
\end{aligned}
$$

It follows from (76) that

$$
\begin{aligned}
\beta(K \beta+r) \bar{I}= & r(K \beta-d) \\
& +\frac{b r(1-m) d_{2}}{a}-\frac{p r r_{1} b^{2}(1-m)^{2}}{a\left(r_{1}+d_{1}\right)} \underline{I}, \\
\beta(K \beta+r) \underline{I}= & r(K \beta-d) \\
& +\frac{b r(1-m) d_{2}}{a}-\frac{p r r_{1} b^{2}(1-m)^{2}}{a\left(r_{1}+d_{1}\right)} \bar{I},
\end{aligned}
$$

and (77) minus (78) results in

$$
\left[\beta(K \beta+r)-\frac{p r r_{1} b^{2}(1-m)^{2}}{a\left(r_{1}+d_{1}\right)}\right](\bar{I}-\underline{I})=0 .
$$

If $a \beta\left(r_{1}+d_{1}\right)(K \beta+r) \neq p r r_{1} b^{2}(1-m)^{2}$, then we derive from (79) that $\bar{I}=I$. It therefore follows from (76) that $\bar{S}=\underline{S}, \bar{Y}_{1}=\underline{Y}_{1}, \bar{Y}_{2}=\underline{Y}_{2}$. We therefore conclude that $E^{*}$ is globally attractive. The proof is complete. 


\section{Conclusion}

In this paper, we have incorporated a prey refuge, stage structure for the predator, and time delay due to the gestation of the predator into an ecoepidemiological predator-prey model. By using Lyapunov functions and the LaSalle invariant principle, the global stability of each of the boundary equilibria of the model is discussed. By using the iteration technique and comparison arguments, sufficient conditions are derived for the global attractivity of the positive equilibrium of the model. By Theorem 4, we see that the predator population go to extinction if $0<\operatorname{pbrr}_{1}(1-m)(K \beta-d)<\beta d_{2}\left(r_{1}+d_{1}\right)(K \beta+$ $r)$. By Theorem 6, we see that if $\operatorname{pbrr}_{1}(1-m)(K \beta-d)>$ $\beta d_{2}\left(r_{1}+d_{1}\right)(K \beta+r)$ and $a \beta\left(r_{1}+d_{1}\right)(K \beta+r) \neq \operatorname{prr}_{1} b^{2}(1-m)^{2}$, then both the prey and the predator species of model (2) are permanent.

\section{Conflict of Interests}

The authors declare that there is no conflict of interests regarding the publication of this paper.

\section{Acknowledgment}

This work was supported by the National Natural Science Foundation of China (11101117).

\section{References}

[1] R. M. Anderso and R. M. May, Intections Disease of Humans Dynamics and Control, Oxford University Press, Oxford, UK, 1991.

[2] S. Wang, The research of eco-epidemiological of models incorporating prey refuges [Ph.D. thesis], Lanzhou University, 2012.

[3] W. Wang and L. Chen, "A predator-prey system with stagestructure for predator," Computers \& Mathematics with Applications, vol. 33, no. 8, pp. 83-91, 1997.

[4] Y. N. Xiao and L. S. Chen, "Global stability of a predator-prey system with stage structure for the predator," Acta Mathematica Sinica, vol. 20, no. 1, pp. 63-70, 2004.

[5] R. Xu and Z. Ma, "Stability and Hopf bifurcation in a predatorprey model with stage structure for the predator," Nonlinear Analysis: Real World Applications, vol. 9, no. 4, pp. 1444-1460, 2008.

[6] W. G. Aiello and H. I. Freedman, "A time-delay model of singlespecies growth with stage structure," Mathematical Biosciences, vol. 101, no. 2, pp. 139-153, 1990.

[7] Y. Kuang and J. W. H. So, "Analysis of a delayed two-stage population model with space-limited recruitment," SIAM Journal on Applied Mathematics, vol. 55, no. 6, pp. 1675-1696, 1995.

[8] J. Hale, Theory of Functional Differential Equations, Springer, Heidelberg, Germany, 1977.

[9] Y. Kuang, Delay Differential Equation with Application in Population Synamic, Academic Press, New York, NY, USA, 1993.

[10] R. Xu and Z. Ma, "Stability and Hopf bifurcation in a predatorprey model with stage structure for the predator," Nonlinear Analysis. Real World Applications, vol. 9, no. 4, pp. 1444-1460, 2008. 


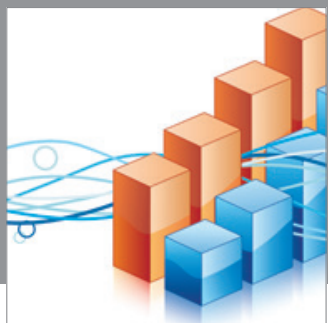

Advances in

Operations Research

mansans

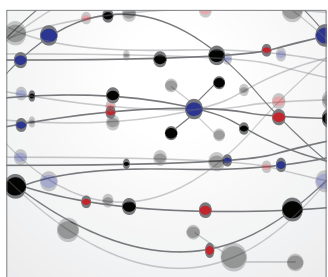

The Scientific World Journal
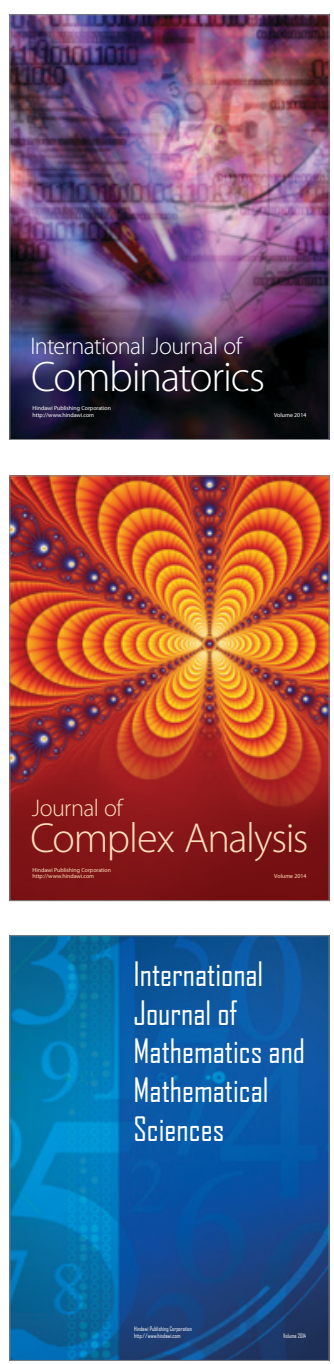
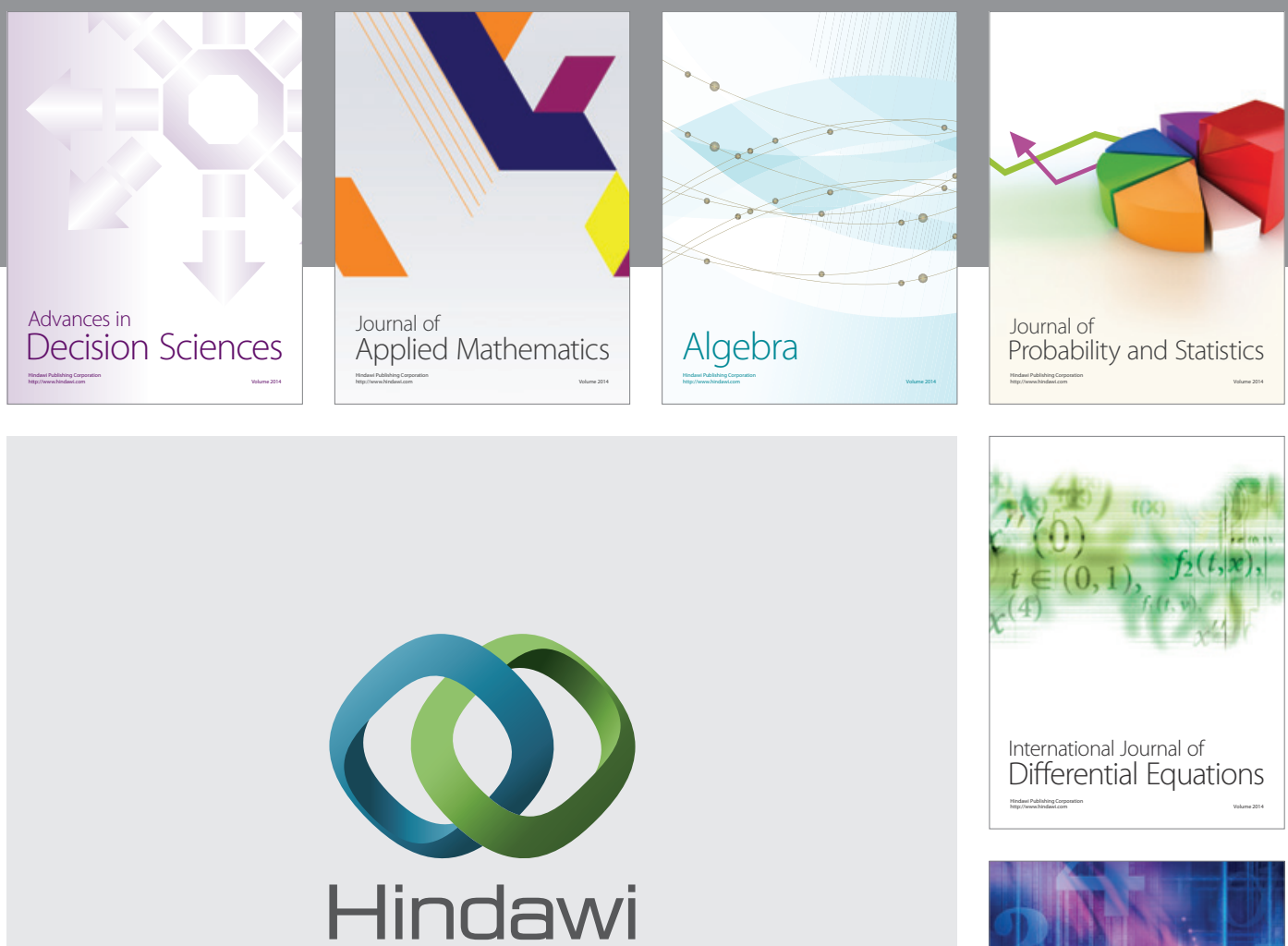

Submit your manuscripts at http://www.hindawi.com
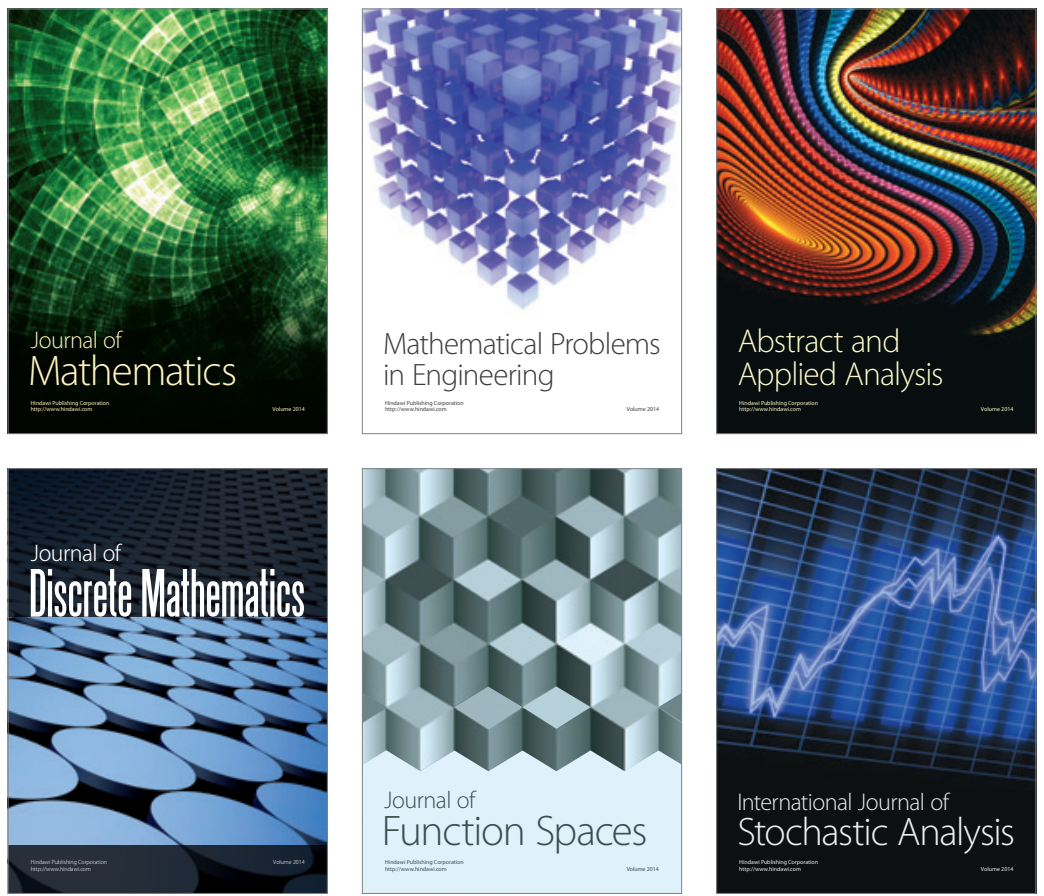

Journal of

Function Spaces

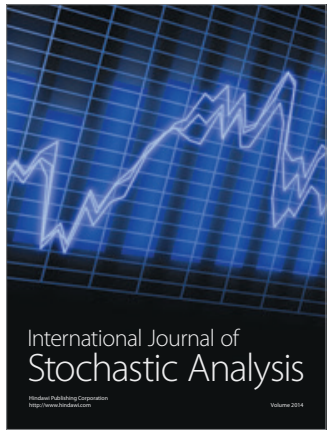

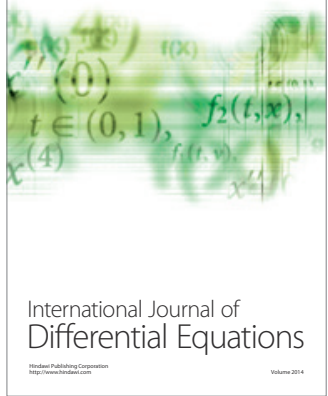
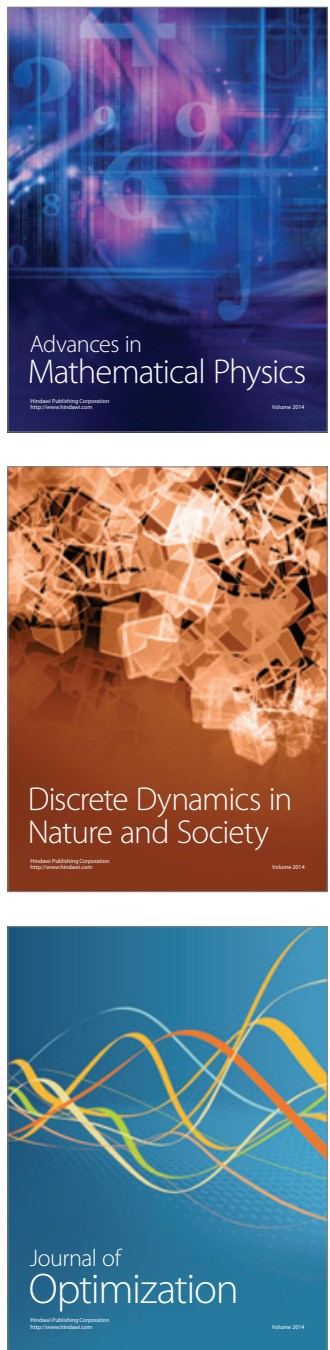